\title{
Best Proximity Points of Multivalued Hardy-Roger's Type (Cyclic) Contractive Mappings of $b$-Metric Spaces
}

\author{
Basit Ali $\mathbb{D}^{1},{ }^{1}$ Arshad Ali Khan $\mathbb{D}^{1},{ }^{1}$ and Azhar Hussain $\mathbb{D}^{2}$ \\ ${ }^{1}$ Department of Mathematics, School of Science (SSc), University of Management and Technology (UMT), C-II, Johar Town, \\ Lahore 54770, Pakistan \\ ${ }^{2}$ Department of Mathematics, University of Sargodha, Sargodha 40100, Pakistan \\ Correspondence should be addressed to Azhar Hussain; azhar.hussain@uos.edu.pk
}

Received 9 December 2021; Accepted 28 December 2021; Published 24 January 2022

Academic Editor: Sun Young Cho

Copyright (c) 2022 Basit Ali et al. This is an open access article distributed under the Creative Commons Attribution License, which permits unrestricted use, distribution, and reproduction in any medium, provided the original work is properly cited.

In this article, we introduce a new type of generalized multivalued Hardy and Roger's type proximal contractive and proximal cyclic contractive mappings of $b$-metric spaces and develop some results for the existence of best proximity point(s). Moreover, we obtain some results for the existence and uniqueness of best proximity points for single-valued mappings. Examples are given to explain the main results.

\section{Introduction}

The metric fixed point theory plays a very fundamental role in many fields of mathematics especially in nonlinear analysis and some related disciplines. The fundamental tool of this theory is the Banach contraction principle (shortly $\mathrm{BCP})[1]$ which states that if a self-mapping $T: P \longrightarrow P$ of a complete metric space $(P, \varrho)$ with metric $\varrho$ satisfies

$$
\varrho\left(T p_{1}, T p_{2}\right) \leq k \varrho\left(p_{1}, p_{2}\right),
$$

for all $p_{1}, p_{2} \in P$, and for some $k \in[0,1), T$ has a unique fixed point, that is, there exists a point $p \in P$, such that

$$
\varrho(p, T p)=0 .
$$

A mapping that satisfies (1) is known as Banach contraction. After this remarkable result, many mathematicians contributed for the development of fixed-point theory by producing many results with different generalized contractive mappings in complete metric spaces, for details one can see [2-8] and the references therein. One of the important generalizations of BCP was presented by Edelstein [9] in 1962. Later on, many mathematicians generalized Edelstein's result, for instance Meir and Keeler [10] in 1969 and Reich [11] in 1971. Reich's result has been further generalized by Hardy and Roger [12] in 1973 as follows.

Theorem 1. Let $(P, \varrho)$ be a metric space and $T: P \longrightarrow P$ a self-mapping satisfying the following conditions for all $p_{1}, p_{2} \in P$ :

(1)

$$
\begin{aligned}
\varrho\left(T p_{1}, T p_{2}\right) \leq & \alpha \varrho\left(p_{1}, T p_{1}\right)+\beta \varrho\left(p_{2}, T p_{2}\right)+\gamma \varrho\left(p_{1}, T p_{2}\right) \\
& +\delta \varrho\left(p_{2}, T p_{1}\right)+\tau \varrho\left(p_{1}, p_{2}\right),
\end{aligned}
$$

where $\alpha, \beta, \gamma, \delta, \tau$ are nonnegative reals.

Set $\Omega=\alpha+\beta+\gamma+\delta+\tau$. Then,

(a) If $P$ is complete and $\Omega<1$, then $T$ has a unique fixed point

(b) If (1) is modified as

$\left(1^{\prime}\right)$ for all $p_{1} \neq p_{2}$ implies

$$
\begin{aligned}
\varrho\left(T p_{1}, T p_{2}\right)< & \alpha \varrho\left(p_{1}, T p_{1}\right)+\beta \varrho\left(p_{2}, T p_{2}\right)+\gamma \varrho\left(p_{1}, T p_{2}\right) \\
& +\delta \varrho\left(p_{2}, T p_{1}\right)+\tau \varrho\left(p_{1}, p_{2}\right),
\end{aligned}
$$


and $P$ is compact, $T$ is continuous, and $\Omega=1$; then, $T$ has a unique fixed point.

Nadler [13] in 1969 generalized the BCP in the context of multivalued mappings of complete metric spaces. Later on, Nadler's result has been generalized by Prolla [14] in 1983.

Meanwhile, the metric space has been generalized to $b$-metric space; by then, the fixed point theory has been further generalized for single-valued and multivalued mappings in the context of $b$-metric space, for instance, Bakhtin [15] in 1989 and Czerwik [16] in 1993.

For nonself mapping, $T: R \longrightarrow S(R$ and $S$ are two nonempty sets), such that $R \cap T(R)=\varnothing$ (empty set); then, it is not possible to find the fixed point of $T$. The best way to deal with such situation is to explore a point $r$ in $R$, such that

$$
\varrho(r, \operatorname{Tr})=\varrho(R, S)
$$

where

$$
\varrho(R, S)=\inf _{r \in R, s \in S} \varrho(r, s),
$$

and if such a point in $R$ exists, it is called the best proximity point of $T$. If $R=S$, then the best proximity point becomes a fixed point. So, best proximity point theory is the proper generalization of fixed-point theory. Fan's result [17] in 1969 was probably the first attempt in this direction.

Later on, many mathematicians extended Fan's result and developed some best proximity point results. For more details, one can see [18]. Best proximity point theory has been further developed by using different proximal contractions, for more details, one can see references [19-23].

Kirk [24] in 2003 introduced cyclic contraction and developed some fixed points results. Later on in 2006, Eldered and Veeramani [25] developed some best proximity point results for cyclic contractions.

Basha in 2019 [21] introduced proximal contractive and proximal cyclic contractive mappings and developed some results for the existence and uniqueness of best proximity point.

Recently, in 2021, Hiranmoy et al. [26] introduced proximal Kannan-type and proximal cyclic Kannan-type contractive mappings in metric spaces (compare with [21]) and developed some best proximity point results.

Motivated by the contractive mappings of Hiranmoy, we introduce the notion of multivalued Hardy and Roger's type proximal and cyclic proximal contractive mappings and develop some results for the existence of best proximity points in $b$-metric space. Furthermore, we give some examples to explain the results.

\section{Preliminaries}

Throughout this article, $\mathbb{R}, \mathbb{R}^{+}, \mathbb{N}, \mathbb{N}_{1}$, and $\wp(P)$ denote the set of reals, nonnegative reals, positive integers, nonnegative integers, and collection of nonempty subsets of $P$, respectively.

Definition 2. Let $P$ be a nonempty set and $b \geq 1$ a real number. The mapping $\varrho_{b}: P \times P \longrightarrow[0, \infty)$ is a $b$-metric and $\left(P, \varrho_{b}\right)$ is called $b$-metric space if $\varrho_{b}$ satisfies the following axioms:

$$
\begin{aligned}
& \left(\mathrm{b}_{1}\right) \varrho_{b}\left(p_{1}, p_{2}\right)=0 \text { if and only if } p_{1}=p_{2} \\
& \left(\mathrm{~b}_{2}\right) \varrho_{b}\left(p_{1}, p_{2}\right)=\varrho_{b}\left(p_{2}, p_{1}\right) \\
& \left(\mathrm{b}_{3}\right) \varrho_{b}\left(p_{1}, p_{2}\right) \leq b\left[\varrho_{b}\left(p_{1}, p_{3}\right)+\varrho_{b}\left(p_{3}, p_{2}\right)\right], \text { for all } \\
& p_{1}, p_{3}, p_{2} \in P .
\end{aligned}
$$

Throughout this paper, $\varrho$ and $\varrho_{b}$ denote metric and $b$-metric, respectively. Now, suppose that $R$ and $S$ are two nonempty subsets of $\left(P, \varrho_{b}\right)$. Define

$$
\begin{aligned}
\varrho_{b}(R, S) & =\inf \left\{\varrho_{b}(r, s): r \in R, s \in S\right\}, \\
R_{0} & =\left\{r \in R: \varrho_{b}(r, s)=\varrho_{b}(R, S) \text { for some } s \in S\right\}, \\
S_{0} & =\left\{s \in S: \varrho_{b}(r, s)=\varrho_{b}(R, S) \text { for some } r \in R\right\} .
\end{aligned}
$$

Definition 3. A $b$-metric space $\left(P, \varrho_{b}\right)$ is boundedly compact if every bounded sequence in $P$ has a convergent subsequence (compare with [27]).

Definition 4 (see [26]). Let $R$ and $S$ be two nonempty subsets of $(P, \varrho)$. A mapping $T: R \longrightarrow S$ is said to be a proximal Kannan-type contractive mapping if

$$
\left.\begin{array}{l}
\varrho\left(r_{1}, \operatorname{Tr}_{3}\right)=\varrho(R, S) \\
\varrho\left(r_{2}, \operatorname{Tr}_{4}\right)=\varrho(R, S) \\
r_{3} \neq r_{4} \\
\varrho\left(r_{1}, \operatorname{Tr}_{3}\right)=\varrho(R, S) \\
\varrho\left(r_{2}, \operatorname{Tr}_{4}\right)=\varrho(R, S) \\
r_{3}=r_{4}
\end{array}\right\} \text { implies } \varrho\left(r_{1}, r_{2}\right)<\frac{1}{2}\left(\varrho\left(r_{1}, r_{3}\right)+\varrho\left(r_{2}, r_{4}\right)\right) \text {, }
$$

hold for all $r_{1}, r_{2}, r_{3}, r_{4} \in R$. 
Definition 5. Let $R$ and $S$ be two nonempty subsets of $\left(P, \varrho_{b}\right)$. Then, a mapping $T: R \cup S \longrightarrow R \cup S$ is said to be cyclic if $T(R) \subset S$ and $T(S) \subset R$ (compare with [26]).
Definition 6 (see [26]). Let $R$ and $S$ be two nonempty subsets of $(P, \varrho)$. A cyclic mapping $T: R \cup S \longrightarrow R \cup S$ is said to be a proximal cyclic Kannan-type contractive mapping if

$$
\left.\begin{array}{l}
\varrho\left(r_{1}, \mathrm{Tr}_{3}\right)=\varrho(R, S) \\
\varrho\left(r_{2}, \mathrm{Tr}_{4}\right)=\varrho(R, S) \\
\varrho\left(r_{3}, r_{4}\right)>\varrho(R, S)
\end{array}\right\} \text { implies } \varrho\left(r_{1}, r_{2}\right)<\frac{1}{2}\left(\varrho\left(r_{1}, r_{3}\right)+\varrho\left(r_{2}, r_{4}\right)\right),
$$

that hold for all $r_{1}, r_{2}, r_{3}, r_{4} \in R$.

In the following, we introduce a compact weak proximal pair in $b$-metric space.

Definition 7. Let $R$ and $S$ be two nonempty subsets of $\left(P, \varrho_{b}\right)$. The pair $(R, S)$ is said to be a compact weak proximal pair if for bounded sequences $\left(r_{n}\right)$ in $R$ and $\left(s_{n}\right)$ in $S$ with $\varrho_{b}\left(r_{n}, s_{n}\right) \longrightarrow \varrho_{b}(R, S)$ as $n \longrightarrow \infty$, the sequences $\left(r_{n}\right)$ and $\left(s_{n}\right)$ have convergent subsequences in $R$ and $S$, respectively (compare with [26]).

Remark 8. Note that if $R=S$ in above definition, then $(R, R)$ is a compact weak proximal pair if and only if $R$ is boundedly compact.

Now, we present a lemma in the context of $b$-metric space (analogous to [[26], Lemma 2.2]) that will be used in the sequel to prove our main results.

Lemma 9. Let $R$ and $S$ be two nonempty subsets of $\left(P, \varrho_{b}\right)$, such that at least one of $R$ and $S$ is bounded, and $(R, S)$ is a compact weak proximal pair. Then, $R_{0} \neq \varnothing$, and hence, so is $S_{0}$.

Proof. As

$$
\varrho_{b}(R, S)=\inf \left\{\varrho_{b}(r, s): r \in R, s \in S\right\},
$$

so for each $n \in N$, there exists $r_{n} \in R$ and $s_{n} \in S$, such that

$$
\varrho_{b}(R, S) \leq \varrho_{b}\left(r_{n}, s_{n}\right)<\varrho_{b}(R, S)+\frac{1}{n} .
$$

Therefore, the sequence $\left(\varrho_{b}\left(r_{n}, s_{n}\right)\right)$ converges to $\varrho_{b}(R, S)$. Now, we assume that $R$ is bounded. So, there exists a positive real number $K$, such that $\varrho_{b}\left(r_{n}, r_{m}\right) \leq K$ for all $n, m \in \mathbb{N}$, so we have

$$
\begin{aligned}
\varrho_{b}\left(s_{n}, s_{m}\right) & \leq b\left(\varrho_{b}\left(s_{n}, r_{n}\right)+\varrho_{b}\left(r_{n}, s_{m}\right)\right) \\
& \leq b\left[\varrho_{b}\left(s_{n}, r_{n}\right)+b\left(\varrho_{b}\left(r_{n}, r_{m}\right)+\varrho_{b}\left(r_{m}, s_{m}\right)\right)\right],
\end{aligned}
$$

which implies

$$
\varrho_{b}\left(s_{n}, s_{m}\right)<b\left[\varrho_{b}(R, S)+1+b\left(K+\varrho_{b}(R, S)+1\right) .\right.
$$

Therefore, $\left(r_{n}\right)$ and $\left(s_{n}\right)$ are bounded sequences. So by compact weak proximality of the pair $(R, S)$, there exist subsequences $\left(r_{n_{k}}\right)$ of $\left(r_{n}\right)$ and $\left(s_{n_{k}}\right)$ of $\left(s_{n}\right)$, such that $\left(r_{n_{k}}\right)$ converges to $r_{\star} \in R$ and $\left(s_{n_{k}}\right)$ converges to $s_{\star} \in S$. Therefore,

$$
\varrho_{b}\left(r_{n_{k}}, s_{n_{k}}\right) \longrightarrow \varrho_{b}\left(r_{\star}, s_{\star}\right) \text { as } k \longrightarrow \infty \text {. }
$$

Thus, we have

$$
\varrho_{b}\left(r_{\star}, s_{\star}\right)=\varrho_{b}(R, S) .
$$

So, $r_{\star} \in R_{0}$ and $s_{\star} \in S_{0}$. Hence, $R_{0} \neq \varnothing$ and $S_{0} \neq \varnothing$. Similarly, if $S$ is bounded, then $R_{0} \neq \varnothing$ and $S_{0} \neq \varnothing$.

Theorem 10 (see [26]). Let $R$ and $S$ be two nonempty subsets of $(P, \varrho)$, such that at least one of $R$ and $S$ is bounded, and $(R, S)$ is a compact weak proximal pair. Let $T: R \longrightarrow S$ be a proximal Kannan-type contractive mapping and assume that

(i) $T\left(R_{0}\right) \subset S_{0}$

(ii) If $\left(r_{n}\right)$ and $\left(s_{n}\right)$ are two bounded sequences in $R$ and $S$, respectively, such that $\left(\varrho\left(r_{n}, s_{n}\right)\right)$ converges to $\varrho(R, S)$, then $\lim _{n \rightarrow \infty} \varrho\left(r_{n}, r_{n+1}\right)=0$.

Then, $T$ has a unique best proximity point in $R$.

Theorem 11 (see [26]). Let $R$ and $S$ be two nonempty subsets of $(P, \varrho)$, such that at least one of $R$ and $S$ is bounded, and $(R, S)$ is a compact weak proximal pair. Let $T: R \cup S \longrightarrow R \cup S$ be a proximal cyclic Kannan-type contractive mapping and assume that the following conditions hold:

(i) $T\left(R_{0}\right) \subset S_{0}$ and $T\left(S_{0}\right) \subset R_{0}$

(ii) If $\left(r_{n}\right)$ and $\left(s_{n}\right)$ are two bounded sequences in $R$ and $S$, respectively, such that $\left(\varrho\left(r_{n}, s_{n}\right)\right)$ converges to $\varrho(R, S)$, then $\lim _{n \rightarrow \infty} \varrho\left(r_{n}, r_{n+1}\right)=0$.

Then, the following conditions hold: 
(a) There exist $r \in R$ and $s \in S$, such that $\varrho(r, \mathrm{Tr})=$ $\varrho(R, S)$ and $\varrho(s, T s)=\varrho(R, S)$

(b) If $r \in R$ and $s \in S$, such that $\varrho(r, \operatorname{Tr})=\varrho(R, S)$ and $\varrho(s, T s)=\varrho(R, S)$, then $\varrho(r, s)=\varrho(R, S)$.

Now, we introduce the notions of a new type of generalized multivalued Hardy and Roger's proximal contractive and proximal cyclic contractive mappings.

Definition 12. Let $R$ and $S$ be two nonempty subsets of $\left(P, \varrho_{b}\right)$. A mapping $T: R \longrightarrow \wp(S)$ is said to be a new type of generalized multivalued Hardy and Roger's proximal contractive mapping if

$$
\left.\begin{array}{l}
\varrho_{b}\left(r_{1}, \operatorname{Tr}_{3}\right)=\varrho_{b}(R, S) \\
\varrho_{b}\left(r_{2}, \operatorname{Tr}_{4}\right)=\varrho_{b}(R, S) \\
r_{3} \neq r_{4}
\end{array}\right\},
$$

implies,

$$
\left.\begin{array}{l}
\varrho_{b}\left(r_{1}, r_{2}\right)<\alpha \varrho_{b}\left(r_{1}, r_{3}\right)+\beta \varrho_{b}\left(r_{2}, r_{4}\right)+\frac{\gamma}{b^{2}} \varrho_{b}\left(r_{3}, r_{4}\right) \\
+\frac{\delta}{b} \varrho_{b}\left(r_{1}, r_{4}\right)+\frac{\tau}{b} \varrho_{b}\left(r_{2}, r_{3}\right) \\
\varrho_{b}\left(r_{1}, \operatorname{Tr}_{3}\right)=\varrho_{b}(R, S) \\
\varrho_{b}\left(r_{2}, \operatorname{Tr}_{4}\right)=\varrho_{b}(R, S) \\
r_{3}=r_{4}
\end{array}\right\},
$$

implies

$$
\begin{aligned}
\varrho_{b}\left(r_{1}, r_{2}\right) \leq & \alpha \varrho_{b}\left(r_{1}, r_{3}\right)+\beta \varrho_{b}\left(r_{2}, r_{3}\right) \\
& +\frac{\delta}{b} \varrho_{b}\left(r_{1}, r_{4}\right)+\frac{\tau}{b} \varrho_{b}\left(r_{2}, r_{3}\right),
\end{aligned}
$$

which hold for all $r_{1}, r_{2}, r_{3}, r_{4} \in R$, where

$$
\alpha+\beta+\gamma+2 \tau=1, \beta \neq 1, \gamma+\delta+\tau<1
$$

$, \alpha, \beta, \gamma, \delta, \tau \in \mathbb{R}^{+}$.

Remark 13. If in the Definition 12, we replace $T: R \longrightarrow \wp(S)$ by $T: R \longrightarrow S$, then $T$ is said to be a new type of generalized Hardy and Roger's proximal contractive mapping.

Definition 14. Let $R$ and $S$ be two nonempty subsets of $\left(P, \varrho_{b}\right)$. A multivalued cyclic mapping $T: R \cup S \longrightarrow$ $\wp(R) \cup \wp(S)$ is said to be a new type of generalized multivalued Hardy and Roger's proximal cyclic contractive mapping if

$$
\left.\begin{array}{l}
\varrho_{b}\left(r_{1}, \operatorname{Tr}_{3}\right)=\varrho_{b}(R, S) \\
\varrho_{b}\left(r_{2}, \operatorname{Tr}_{4}\right)=\varrho_{b}(R, S) \\
\varrho_{b}\left(r_{3}, r_{4}\right)>\varrho_{b}(R, S)
\end{array}\right\},
$$

implies,

$$
\left.\begin{array}{l}
\varrho_{b}\left(r_{1}, r_{2}\right)<\alpha \varrho_{b}\left(r_{1}, r_{3}\right)+\beta \varrho_{b}\left(r_{2}, r_{4}\right)+\frac{\gamma}{b^{2}} \varrho_{b}\left(r_{3}, r_{4}\right) \\
+\frac{\delta}{b} \varrho_{b}\left(r_{1}, r_{4}\right)+\frac{\tau}{b} \varrho_{b}\left(r_{2}, r_{3}\right) \\
\varrho_{b}\left(r_{1}, \operatorname{Tr}_{3}\right)=\varrho_{b}(R, S) \\
\varrho_{b}\left(r_{2}, \operatorname{Tr}_{4}\right)=\varrho_{b}(R, S) \\
\varrho_{b}\left(r_{3}, r_{4}\right)=\varrho_{b}(R, S)
\end{array}\right\} \text { implies } \varrho_{b}\left(r_{1}, r_{2}\right)=\varrho_{b}(R, S),
$$

which hold for all $r_{1}, r_{2}, r_{3}, r_{4} \in R$, where

$$
\alpha+\beta+\gamma+2 \tau=1, \alpha \neq 1, \beta \neq 1, \gamma+\delta+\tau<1, \alpha, \beta, \gamma, \delta, \tau \in \mathbb{R}^{+} .
$$

Remark 15. If in the Definition 14, we replace $T: R \cup S \longrightarrow \wp(R) \cup \wp(S)$ by $T: R \cup S \longrightarrow R \cup S$, then $T$ is said to be a new type of generalized Hardy and Roger's proximal cyclic contractive mapping.

\section{Best Proximity Points Results for a New Type of Multivalued Hardy and Roger's Proximal Contractive Mappings in $b$-metric Space}

The following is our main result of this section.

Theorem 16. Let $R$ and $S$ be two nonempty subsets of $\left(P, \varrho_{b}\right)$, such that at least one of $R$ and $S$ is bounded and $(R, S)$ is a compact weak proximal pair. Let $T: R \longrightarrow \wp(S)$ be a new type of generalized multivalued Hardy and Roger's proximal contractive mapping. Further assume that

(i) For each $r \in R_{0}$, $\operatorname{Tr} \subset S_{0}$

(ii) If $\left(r_{n}\right)$ and $\left(s_{n}\right)$ are two bounded sequences in $R$ and $S$, respectively, such that $\left(\varrho_{b}\left(r_{n}, s_{n}\right)\right)$ converges to $\varrho_{b}(R, S)$, then $\lim _{n \longrightarrow \infty} \varrho_{b}\left(r_{n}, r_{n+1}\right)=0$.

Then, $T$ has a best proximity point.

Proof. Lemma 9 implies $R_{0} \neq \varnothing$. Let $r_{0} \in R_{0}$; then, $\operatorname{Tr}_{0} \subset S_{0}$. We can pick an element $s_{1} \in \operatorname{Tr}_{0} \subset S_{0}$, so that there exists $r_{1} \in R$, such that 


$$
\varrho_{b}\left(r_{1}, s_{1}\right)=\varrho_{b}(R, S) .
$$

Continuing this way, we can construct sequences $\left(r_{n}\right)$ in $R$ and $\left(s_{n}\right)$ in $T r_{n-1}$, such that

$$
\varrho_{b}\left(r_{n}, s_{n}\right)=\varrho_{b}(R, S),
$$

for all $n \in \mathbb{N}$. Therefore,

$$
\varrho_{b}(R, S) \leq \varrho_{b}\left(r_{n}, \operatorname{Tr}_{n-1}\right) \leq \varrho_{b}\left(r_{n}, s_{n}\right)=\varrho_{b}(R, S),
$$

that is,

$$
\varrho_{b}\left(r_{n}, T r_{n-1}\right)=\varrho_{b}(R, S)
$$

If $r_{n}=r_{n-1}$ for some $n \in \mathbb{N}$, then $r_{n-1}$ is the best proximity point of $T$, and the proof is completed. So, we may assume that $r_{n} \neq r_{n-1}$ for all $n \in \mathbb{N}$. Now, we show that $\left(r_{n}\right)$ and $\left(s_{n}\right)$ are bounded sequences. As we have

$$
\begin{aligned}
\varrho_{b}\left(r_{n}, T r_{n-1}\right) & =\varrho_{b}(R, S), \\
\varrho_{b}\left(r_{n+1}, \operatorname{Tr}_{n}\right) & =\varrho_{b}(R, S), \\
r_{n} & \neq r_{n-1},
\end{aligned}
$$

so by the given condition, we obtain

$$
\begin{aligned}
\varrho_{b}\left(r_{n}, r_{n+1}\right)< & \alpha \varrho_{b}\left(r_{n}, r_{n-1}\right)+\beta \varrho_{b}\left(r_{n+1}, r_{n}\right)+\frac{\gamma}{b^{2}} \varrho_{b}\left(r_{n-1}, r_{n}\right), \\
& +\frac{\delta}{b} \varrho_{b}\left(r_{n}, r_{n}\right)+\frac{\tau}{b} \varrho_{b}\left(r_{n+1}, r_{n-1}\right) \\
\leq & \alpha \varrho_{b}\left(r_{n}, r_{n-1}\right)+\beta \varrho_{b}\left(r_{n+1}, r_{n}\right) \\
& +\gamma \varrho_{b}\left(r_{n-1}, r_{n}\right)+\tau\left(\varrho_{b}\left(r_{n-1}, r_{n}\right)+\varrho_{b}\left(r_{n}, r_{n+1}\right)\right),
\end{aligned}
$$

which implies

$$
(1-(\beta+\tau)) \varrho_{b}\left(r_{n}, r_{n+1}\right)<(\alpha+\gamma+\tau) \varrho_{b}\left(r_{n-1}, r_{n}\right) .
$$

If $1-(\beta+\tau)=0$, then $\beta+\tau=1$, so (17) implies $\alpha=\gamma=\tau=0$, and so $\beta=1$, a contradiction. Therefore, $1-$ $(\beta+\tau) \neq 0$ and $1-(\beta+\tau)=\alpha+\gamma+\tau$. Hence, we get

$$
\varrho_{b}\left(r_{n}, r_{n+1}\right)<\varrho_{b}\left(r_{n-1}, r_{n}\right),
$$

that is,

$\varrho_{b}\left(r_{n}, r_{n+1}\right)<\varrho_{b}\left(r_{n-1}, r_{n}\right)<\cdots<\varrho_{b}\left(r_{0}, r_{1}\right)=K$ (say).

As

$$
\begin{aligned}
\varrho_{b}\left(r_{n}, T r_{n-1}\right) & =\varrho_{b}(R, S), \\
\varrho_{b}\left(r_{m}, T r_{m-1}\right) & =\varrho_{b}(R, S),
\end{aligned}
$$

so, if $r_{n-1}=r_{m-1}$, then we have

$$
\begin{aligned}
\varrho_{b}\left(r_{n}, r_{m}\right) \leq & b\left(\varrho_{b}\left(r_{n}, r_{m-1}\right)+\varrho_{b}\left(r_{m-1}, r_{m}\right)\right), \\
\leq & b\left[b\left(\varrho_{b}\left(r_{n}, r_{n-1}\right)+\varrho_{b}\left(r_{n-1}, r_{m-1}\right)\right)\right. \\
& \left.+\varrho_{b}\left(r_{m-1}, r_{m}\right)\right], \\
\leq & \left(b^{2}+b\right) K .
\end{aligned}
$$

If $r_{n-1} \neq r_{m-1}$, then

$$
\begin{aligned}
\varrho_{b}\left(r_{n}, r_{m}\right)< & \alpha \varrho_{b}\left(r_{n}, r_{n-1}\right)+\beta \varrho_{b}\left(r_{m}, r_{m-1}\right)+\frac{\gamma}{b^{2}} \varrho_{b}\left(r_{n-1}, r_{m-1}\right), \\
& +\frac{\delta}{b} \varrho_{b}\left(r_{n}, r_{m-1}\right)+\frac{\tau}{b} \varrho_{b}\left(r_{m}, r_{n-1}\right) \\
\leq & (\alpha+\beta) K+\frac{\gamma}{b^{2}}\left[b\left(\varrho_{b}\left(r_{n-1}, r_{n}\right)+b\left(\varrho_{b}\left(r_{n}, r_{m}\right)+\varrho_{b}\left(r_{m}, r_{m-1}\right)\right)\right)\right] \\
& +\delta\left(\varrho_{b}\left(r_{n}, r_{m}\right)+\varrho_{b}\left(r_{m}, r_{m-1}\right)\right)+\tau\left(\varrho_{b}\left(r_{m}, r_{n}\right)+\varrho_{b}\left(r_{n}, r_{n-1}\right)\right),
\end{aligned}
$$

which implies

$$
\left(1-(\gamma+\delta+\tau) \varrho_{b}\left(r_{n}, r_{m}\right)<(\alpha+\beta+2 \gamma+\delta+\tau) K\right),
$$

and by (2), $\gamma+\delta+\tau<1$. Therefore,

$$
\varrho_{b}\left(r_{n}, r_{m}\right)<\frac{(\alpha+\beta+\gamma+\delta+\tau)}{1-(\gamma+\delta+\tau)} K .
$$

Hence, $\left(r_{n}\right)$ is a bounded sequence. Furthermore, for all $n, m \in \mathbb{N}$, we have

$$
\begin{aligned}
\varrho_{b}\left(s_{n}, s_{m}\right) & \leq b\left(\varrho_{b}\left(s_{n}, r_{n}\right)+\varrho_{b}\left(r_{n}, s_{m}\right)\right) \\
& \leq b \varrho_{b}(R, S)+b^{2}\left(\varrho_{b}\left(r_{n}, r_{m}\right)+\varrho_{b}\left(r_{m}, s_{m}\right)\right) \\
& \leq\left(b+b^{2}\right) \varrho_{b}(R, S)+b^{2} \frac{(\alpha+\beta+\gamma+\delta+\tau)}{1-(\gamma+\delta+\tau)} K .
\end{aligned}
$$

Therefore, $\left(s_{n}\right)$ is also a bounded sequence. From (69), it is clear that $\left(\varrho_{b}\left(r_{n}, r_{n+1}\right)\right)$ is a decreasing sequence of 
nonnegative real numbers and hence convergent. Using hypothesis (ii), $\left(\varrho_{b}\left(r_{n}, r_{n+1}\right)\right)$ converges to 0 . Now, by compact weak proximality of the pair $(R, S)$, there exist two subsequences $\left(r_{n_{k}}\right)$ of $\left(r_{n}\right)$ and $\left(s_{n_{k}}\right)$ of $\left(s_{n}\right)$, such that $\left(r_{n_{k}}\right)$ converges to some $r_{\star} \in R$ and $\left(s_{n_{k}}\right)$ converges to some $s_{\star} \in S$. Consequently,

$$
\varrho_{b}\left(r_{n_{k}}, s_{n_{k}}\right) \longrightarrow \varrho_{b}\left(r_{\star}, s_{\star}\right) \text { as } k \longrightarrow \infty .
$$

Consequently,

$$
\varrho_{b}\left(r_{\star}, s_{\star}\right)=\varrho_{b}(R, S) .
$$

Thus, $r_{\star} \in R_{0}$, which implies $T r_{\star} \subset S_{0}$. For each $v \in T r_{\star}$, there exists $v \in R$, such that $\varrho_{b}(v, v)=\varrho_{b}(R, S)$. Now,

$$
\varrho_{b}(R, S) \leq \varrho_{b}\left(v, T r_{\star}\right) \leq \varrho_{b}(v, v)=\varrho_{b}(R, S),
$$

which implies

$$
\varrho_{b}\left(v, T r_{\star}\right)=\varrho_{b}(R, S) .
$$

Moreover, we have

$$
\varrho_{b}\left(r_{n_{k}+1}, r_{\star}\right) \leq b\left(\varrho_{b}\left(r_{n_{k}+1}, r_{n_{k}}\right)+\varrho_{b}\left(r_{n_{k}}, r_{\star}\right)\right) .
$$

Letting $k \longrightarrow \infty$, we get

$$
\lim _{k \rightarrow \infty} r_{n_{k}+1}=r_{\star},
$$

then using the facts

$$
\begin{aligned}
\varrho_{b}\left(r_{n_{k}+1}, T r_{n_{k}}\right) & =\varrho_{b}(R, S), \\
\varrho_{b}\left(v, T r_{\star}\right) & =\varrho_{b}(R, S),
\end{aligned}
$$

we get

$$
\begin{aligned}
\varrho_{b}\left(r_{n_{k}+1}, v\right) \leq & \alpha \varrho_{b}\left(r_{n_{k}+1}, r_{n_{k}}\right)+\beta \varrho_{b}\left(v, r_{\star}\right)+\frac{\gamma}{b^{2}} \varrho_{b}\left(r_{n_{k}}, r_{\star}\right) \\
& +\frac{\delta}{b} \varrho_{b}\left(r_{n_{k}+1}, r_{\star}\right)+\frac{\tau}{b} \varrho_{b}\left(v, r_{n_{k}}\right) \\
\leq & \alpha \varrho_{b}\left(r_{n_{k}+1}, r_{n_{k}}\right)+\beta \varrho_{b}\left(v, r_{\star}\right)+\gamma \varrho_{b}\left(r_{n_{k}}, r_{\star}\right) \\
& +\delta \varrho_{b}\left(r_{n_{k}+1}, r_{\star}\right)+\tau \varrho_{b}\left(v, r_{n_{k}}\right) .
\end{aligned}
$$

Letting $k \longrightarrow \infty$, we get

$$
[1-(\beta+\tau)] \varrho_{b}\left(v, r_{\star}\right) \leq 0 .
$$

It implies $v=r_{\star}$. Thus, we have $\varrho_{b}\left(r_{\star}, T r_{\star}\right)=\varrho_{b}(R, S)$, that is, $r_{\star}$ is a best proximity point of $T$. This completes the proof.

Now, we give an example to explain our claim.

Example 17. Let $P=\mathbb{R}, R=[1,2]$, and $S=[1 / 4,1 / 2]$. Consider

$$
\varrho_{b}\left(p_{1}, p_{2}\right)=\left|p_{1}-p_{2}\right|^{2}
$$

for all $p_{1}, p_{2} \in \mathbb{R}$. Then, $\varrho_{b}$ is a $b$-metric on $P$ with $b=2$. It implies $\varrho_{b}(R, S)=\{1 / 4\}, R_{0}=\{1\}$, and $S_{0}=\{1 / 2\}$; now, define a mapping $T: R \longrightarrow \wp(S)$ as follows:

$$
\operatorname{Tr}=\left\{\frac{1}{2^{n}}: 1 \leq n \leq r\right\} .
$$

It implies for each $r \in R_{0}, \operatorname{Tr} \subset S_{0}$. Now, we check $T$ is a new type of generalized multivalued Hardy and Roger's proximal contractive mapping. Let

$$
r_{1}, r_{2}, r_{3}, r_{4} \in R
$$

Then, we discuss two possible cases.

Case 1: if

$$
r_{1}, r_{2}, r_{3}, r_{4}>1 \text {, }
$$

then

$$
\begin{aligned}
& \varrho_{b}\left(r_{1}, \operatorname{Tr}_{3}\right) \neq d(R, S), \\
& \varrho_{b}\left(r_{2}, \operatorname{Tr}_{4}\right) \neq d(R, S) .
\end{aligned}
$$

Case 2: if

$$
r_{1}=r_{2}=r_{3}=r_{4}=1 \text {, }
$$

then

$$
\begin{aligned}
\varrho_{b}\left(r_{1}, \operatorname{Tr}_{3}\right) & =d(R, S), \\
\varrho_{b}\left(r_{2}, \operatorname{Tr}_{4}\right) & =d(R, S), \\
r_{3} & =r_{4} .
\end{aligned}
$$

It implies

$$
\begin{aligned}
\varrho_{b}\left(r_{1}, r_{2}\right)= & 0 \leq \alpha \varrho_{b}\left(r_{1}, r_{3}\right)+\beta \varrho_{b}\left(r_{2}, r_{4}\right)+\frac{\delta}{b} \varrho_{b}\left(r_{1}, r_{4}\right) \\
& +\frac{\tau}{b} \varrho_{b}\left(r_{2}, r_{3}\right),
\end{aligned}
$$

where

$$
\alpha+\beta+\gamma+2 \tau=1, \alpha \neq 1, \beta \neq 1, \gamma+\delta+\tau<1,
$$

$\alpha, \beta, \gamma, \delta, \tau \in \mathbb{R}^{+}$. So, all axioms of Theorem 16 are satisfied. Hence, $T$ has the best proximity points set $\{1\}$.

Theorem 18. Let $R$ and $S$ be two nonempty subsets of $\left(P, \varrho_{b}\right)$, such that at least one of $R$ and $S$ is bounded, and $(R, S)$ is a compact weak proximal pair. Let $T: R \longrightarrow S$ be a new type of generalized Hardy and Roger's proximal contractive mapping and assume that

(i) For each $r \in R_{0}, \operatorname{Tr} \in S_{0}$

(ii) If $\left(r_{n}\right)$ and $\left(s_{n}\right)$ are two bounded sequences in $R$ and $S$, respectively, such that $\left(\varrho_{b}\left(r_{n}, s_{n}\right)\right)$ converges to $\varrho_{b}(R, S)$, then $\lim _{n \longrightarrow \infty} \varrho_{b}\left(r_{n}, r_{n+1}\right)=0$

Then, $T$ has a unique best proximity point. 
Proof. Existence of best proximity point follows from Theorem 16. Now, to prove the uniqueness, consider $r_{1}$ and $r_{2}$ be two distinct best proximity points of $T$. Then, we have

$$
\begin{aligned}
\varrho_{b}\left(r_{1}, \operatorname{Tr}_{1}\right) & =\varrho_{b}(R, S), \\
\varrho_{b}\left(r_{2}, \operatorname{Tr}_{2}\right) & =\varrho_{b}(R, S), \\
r_{1} & \neq r_{2} .
\end{aligned}
$$

It implies

$$
\begin{aligned}
\varrho_{b}\left(r_{1}, r_{2}\right) & <\alpha \varrho_{b}\left(r_{1}, r_{1}\right)+\beta \varrho_{b}\left(r_{2}, r_{2}\right)+\frac{\gamma}{b^{2}} \varrho_{b}\left(r_{1}, r_{2}\right)+\frac{\delta}{b} \varrho_{b}\left(r_{1}, r_{2}\right)+\frac{\tau}{b} \varrho_{b}\left(r_{1}, r_{2}\right), \\
& \leq \alpha \varrho_{b}\left(r_{1}, r_{1}\right)+\beta \varrho_{b}\left(r_{2}, r_{2}\right)+\gamma \varrho_{b}\left(r_{1}, r_{2}\right)+\delta \varrho_{b}\left(r_{1}, r_{2}\right)+\tau \varrho_{b}\left(r_{1}, r_{2}\right),
\end{aligned}
$$

so

$$
\varrho_{b}\left(r_{1}, r_{2}\right)<(\gamma+\delta+\tau) \varrho_{b}\left(r_{1}, r_{2}\right)<\varrho_{b}\left(r_{1}, r_{2}\right),
$$

a contradiction as $\gamma+\delta+\tau<1$. Hence, $T$ has a unique best proximity point.

Corollary 19. If we take in Theorem $18 b=1$ and $\alpha=\beta=1 / 2, \gamma=\delta=\tau=0$, then we get Theorem 10 .

\section{Best Proximity Points Results for a New Type of Multivalued Hardy and Roger's Proximal Cyclic Contractive Mappings in $b$-metric Space}

In this section, we consider new type of multivalued Hardy and Roger's proximal cyclic contractive mapping for the existence of best proximity points.

Theorem 20. Let $R$ and $S$ be two nonempty subsets of $\left(P, \varrho_{b}\right)$, such that at least one of $R$ and $S$ is a bounded subset of $P$ and $(R, S)$ is a compact weak proximal pair. Let $T: R \cup S \longrightarrow \wp(R) \cup \wp(S)$ be a new type of generalized multivalued Hardy and Roger's proximal cyclic contractive mapping and assume that

(i) For each $r \in R_{0}$, $T s \subset S_{0}$, and for each $s \in S_{0}$, Ts $\subset R_{0}$, and

(ii) If $\left(r_{n}\right)$ and $\left(s_{n}\right)$ are two bounded sequences in $R$ and $S$, respectively, such that $\left(\varrho_{b}\left(r_{n}, s_{n}\right)\right)$ converges to $\varrho_{b}(R, S)$, then $\lim _{n \longrightarrow \infty} \varrho_{b}\left(r_{n}, r_{n+1}\right)=0$.

Then, there exist $r \in R$ and $s \in S$, such that $\mathrm{\varrho}_{b}(r, \operatorname{Tr})=$ $\varrho_{b}(R, S)$ and $\varrho_{b}(s, T s)=\varrho_{b}(R, S)$, and furthermore, $\varrho_{b}(r, s)=\varrho_{b}(R, S)$.

Proof. Since $(R, S)$ is a compact weakly proximal pair and at least one of $R$ and $S$ is bounded, so by Lemma 9, it follows that $R_{0} \neq 0$ and $S_{0} \neq 0$. Let $r_{0} \in R_{0}$ and $s_{0} \in S_{0}$ imply $\operatorname{Tr}_{0} \subset S_{0}$ and $T s_{0} \subset R_{0}$, so there exists $r_{1} \in R$ and $s_{1} \in S$, such that

$$
\varrho_{b}\left(r_{1}, v_{1}\right)=\varrho_{b}(R, S)
$$

$v_{1} \in \operatorname{Tr}_{0}$. Continuing this way, we construct sequences $\left(r_{n}\right)$ in $R,\left(s_{n}\right)$ in $S,\left(v_{n}\right)$ in $T r_{n-1}$, and $\left(v_{n}\right)$ in $T s_{n-1}$, such that

$$
\varrho_{b}\left(r_{n}, v_{n}\right)=\varrho_{b}(R, S)
$$

and

$$
\varrho_{b}\left(s_{n}, v_{n}\right)=\varrho_{b}(R, S) .
$$

It implies

$$
\varrho_{b}(R, S) \leq \varrho_{b}\left(r_{n}, \operatorname{Tr}_{n-1}\right) \leq \varrho_{b}\left(r_{n}, v_{n}\right)=\varrho_{b}(R, S),
$$

and

$$
\varrho_{b}(R, S) \leq \varrho_{b}\left(s_{n}, T s_{n-1}\right) \leq \varrho_{b}\left(s_{n}, v_{n}\right)=\varrho_{b}(R, S),
$$

for all $n \in \mathbb{N}$. Thus, we have

$$
\begin{aligned}
\varrho_{b}\left(r_{n}, T r_{n-1}\right) & =\varrho_{b}(R, S), \\
\varrho_{b}\left(s_{n}, T s_{n-1}\right) & =\varrho_{b}(R, S) .
\end{aligned}
$$

First, we assume that $R$ is bounded. Then, there exists a positive real number $K$, such that $\varrho_{b}\left(r_{n}, r_{m}\right) \leq K$ for all $n, m \in \mathbb{N}$. Therefore, for all $n, m \in \mathbb{N}$, we have

$$
\begin{aligned}
\varrho_{b}\left(v_{n}, v_{m}\right) & \leq b\left(\varrho_{b}\left(v_{n}, r_{n}\right)+\varrho_{b}\left(r_{n}, v_{m}\right)\right), \\
& \leq b\left(\varrho_{b}(R, S)+b\left(\varrho_{b}\left(r_{n}, r_{m}\right)+\varrho_{b}\left(r_{m}, v_{m}\right)\right)\right), \\
& \leq b\left(\varrho_{b}(R, S)+b K+b \varrho_{b}(R, S)\right),
\end{aligned}
$$

implies

$$
\varrho_{b}\left(v_{n}, v_{m}\right) \leq\left(b+b^{2}\right) \varrho_{b}(R, S)+b^{2} K
$$

Therefore, $\left(v_{n}\right)$ is bounded. Also, $T$ is cyclic, so for each $s \in S$, Ts $\subset R$, and so $v_{n} \in R$, for all $n \in \mathbb{N}$. Therefore, there exists a positive real number $K_{1}$, such that $\varrho_{b}\left(v_{n}, v_{m}\right) \leq K_{1}$. It implies $\left(v_{n}\right)$ is bounded, so

$$
\begin{aligned}
\varrho_{b}\left(s_{n}, s_{m}\right) & \leq b\left(\varrho_{b}\left(s_{n}, v_{n}\right)+\varrho_{b}\left(v_{n}, s_{m}\right)\right), \\
& \leq b\left(\varrho_{b}(R, S)+b\left(\varrho_{b}\left(v_{n}, v_{m}\right)+\varrho_{b}\left(v_{m}, s_{m}\right)\right)\right), \\
& \leq\left(b+b^{2}\right) \varrho_{b}(R, S)+b^{2} K_{1} .
\end{aligned}
$$


It implies $\left(s_{n}\right)$ is bounded. Thus, $\left(r_{n}\right),\left(s_{n}\right),\left(v_{n}\right)$, and $\left(v_{n}\right)$ all are bounded sequences. On a similar line, we can prove $\left(r_{n}\right),\left(s_{n}\right),\left(v_{n}\right)$, and $\left(v_{n}\right)$ are bounded whenever $S$ is bounded. Since $(R, S)$ is a compact weak pair, therefore, there exist subsequences $\left(r_{n_{k}}\right),\left(s_{n_{k}}\right),\left(v_{n_{k}}\right)$, and $\left(v_{n_{k}}\right)$ of $\left(r_{n}\right)$, $\left(s_{n}\right),\left(v_{n}\right)$, and $\left(v_{n}\right)$, respectively, such that $r_{n_{k}} \longrightarrow r_{\star} \in R$, $s_{n_{k}} \longrightarrow s_{\star} \in S, \quad v_{n_{k}} \longrightarrow v_{\star} \in S$, and $\quad v_{n_{k}} \longrightarrow v_{\star} \in R$, as $k \longrightarrow \infty$. First, we show that $\varrho_{b}\left(r_{\star}, T r_{\star}\right)=\varrho_{b}(R, S)$. As we have

$$
\varrho_{b}\left(r_{n}, T r_{n-1}\right)=\varrho_{b}(R, S),
$$

and

$$
\varrho_{b}\left(r_{n+1}, \operatorname{Tr}_{n}\right)=\varrho_{b}(R, S),
$$

so if $\varrho_{b}\left(r_{n-1}, r_{n}\right)>\varrho_{b}(R, S)$, then we have

$$
\begin{aligned}
& \varrho_{b}\left(r_{n}, r_{n+1}\right)< \alpha \varrho_{b}\left(r_{n}, r_{n-1}\right)+\beta \varrho_{b}\left(r_{n+1}, r_{n}\right)+\frac{\gamma}{b^{2}} \varrho_{b}\left(r_{n-1}, r_{n}\right) \\
&+\frac{\delta}{b} \varrho_{b}\left(r_{n}, r_{n}\right)+\frac{\tau}{b} \varrho_{b}\left(r_{n+1}, r_{n-1}\right), \\
& \leq \alpha \varrho_{b}\left(r_{n}, r_{n-1}\right)+\beta \varrho_{b}\left(r_{n+1}, r_{n}\right)+\gamma \varrho_{b}\left(r_{n-1}, r_{n}\right)+\tau\left(\varrho_{b}\left(r_{n-1}, r_{n}\right)+\varrho_{b}\left(r_{n}, r_{n+1}\right)\right) \\
& \leq(\alpha+\gamma) \varrho_{b}\left(r_{n-1}, r_{n}\right)+\beta \varrho_{b}\left(r_{n}, r_{n+1}\right)+\tau\left(\varrho_{b}\left(r_{n-1}, r_{n}\right)+\varrho_{b}\left(r_{n}, r_{n+1}\right)\right) . \\
& \varrho_{b}\left(v, r_{\star}\right)=\varrho_{b}(R, S) .
\end{aligned}
$$

So,$$
(1-(\beta+\tau)) \varrho_{b}\left(r_{n}, r_{n+1}\right)<(\alpha+\gamma+\tau) \varrho_{b}\left(r_{n-1}, r_{n}\right) .
$$

If $1-(\beta+\tau)=0$, then $\beta+\tau=1$, so (19) implies $\alpha=\gamma=\delta=\tau=0$, and $\beta=1$ is a contradiction. Therefore,

$$
\varrho_{b}\left(r_{n}, r_{n+1}\right)<\varrho_{b}\left(r_{n-1}, r_{n}\right) .
$$

If

$$
\varrho_{b}\left(r_{n-1}, r_{n}\right)=\varrho_{b}(R, S),
$$

then

$$
\varrho_{b}\left(r_{n}, r_{n+1}\right)=\varrho_{b}\left(r_{n-1}, r_{n}\right) .
$$

Therefore,

$$
\varrho_{b}\left(r_{n}, r_{n+1}\right) \leq \varrho_{b}\left(r_{n-1}, r_{n}\right),
$$

for all $n \in \mathbb{N}$, and hence, the sequence $\left(\varrho_{b}\left(r_{n}, r_{n+1}\right)\right)$ is a convergent sequence of real numbers. By hypothesis (ii), it follows that $\left(\varrho_{b}\left(r_{n}, r_{n+1}\right)\right)$ converges to 0 . Now,

$$
\begin{aligned}
\varrho_{b}\left(r_{n_{k}+1}, r_{\star}\right) \leq & b\left(\varrho_{b}\left(r_{n_{k}+1}, r_{n_{k}}\right)+\varrho_{b}\left(r_{n_{k}}, r_{\star}\right)\right) \\
& \longrightarrow 0 \text { as } k \longrightarrow \infty
\end{aligned}
$$

Therefore, $\quad \lim _{k \rightarrow \infty} r_{n_{k}+1}=r_{\star}$. Again, we have $\varrho_{b}\left(r_{n_{k}}, v_{n_{k}}\right) \longrightarrow \varrho_{b}\left(r_{\star}, v_{\star}\right)$ as $k \longrightarrow \infty$, and hence, we get

$$
\varrho_{b}\left(r_{\star}, v_{\star}\right)=\varrho_{b}(R, S) \text {. }
$$

So, $r_{\star} \in R_{0}$ implies $T r_{\star} \subset S_{0}$. Thus, there exists $v \in R$, such that

$$
\varrho_{b}(v, v)=\varrho_{b}(R, S),
$$

where $v \in T r_{\star}$. It implies
If

$$
\varrho_{b}\left(r_{n_{k}}, r_{\star}\right)=\varrho_{b}(R, S),
$$

only for finitely many $k$, then we can exclude those $r_{n_{k}}$ from $\left(r_{n_{k}}\right)$ and then assume

$$
\varrho_{b}\left(r_{n_{k}}, r_{\star}\right)>\varrho_{b}(R, S)
$$

for all $k$. If

$$
\varrho_{b}\left(r_{n_{k}}, r_{\star}\right)=\varrho_{b}(R, S),
$$

for infinitely many $k$, then we can extract a subsequence $\left(r_{n_{k_{1}}}\right)$ from $\left(r_{n_{k}}\right)$, such that

$$
\varrho_{b}\left(r_{n_{k_{l}}}, r_{\star}\right)=\varrho_{b}(R, S),
$$

for all $l$. This gives

$$
\lim _{l \longrightarrow \infty} \varrho_{b}\left(r_{n_{k_{l}}}, r_{\star}\right)=\varrho_{b}(R, S) \text { implies } \varrho_{b}(R, S)=0 .
$$

From the relations

$$
\left.\begin{array}{l}
\varrho_{b}\left(v, T r_{\star}\right)=\varrho_{b}(R, S), \\
\varrho_{b}\left(r_{n_{k_{l}}+1}, T r_{n_{k_{l}}}\right)=\varrho_{b}(R, S),
\end{array}\right\}
$$

and

$$
\varrho_{b}\left(r_{n_{k_{l}}}, r_{\star}\right)=\varrho_{b}(R, S),
$$

we get

$$
\varrho_{b}\left(r_{n_{k_{l}+1}}, v\right)=\varrho_{b}(R, S)=0,
$$

for all $l$. Taking limit as $l \longrightarrow \infty$, we get 


$$
\varrho_{b}\left(r_{\star}, v\right)=0,
$$

so $r_{\star}=v$. Therefore, we have

$$
\varrho_{b}\left(r_{\star}, T r_{\star}\right)=\varrho_{b}(R, S) .
$$

Next, we assume that

$$
\varrho_{b}\left(r_{n_{k_{l}}}, r_{\star}\right)>\varrho_{b}(R, S),
$$

for all $k$; then, from relations

$$
\left.\begin{array}{l}
\varrho_{b}\left(v, T r_{\star}\right)=\varrho_{b}(R, S), \\
\varrho_{b}\left(r_{n_{k_{l}}+1}, T r_{n_{k_{l}}}\right)=\varrho_{b}(R, S),
\end{array}\right\}
$$

we get

$$
\begin{aligned}
\varrho_{b}\left(r_{n_{k_{l}}+1}, v\right)< & \alpha \varrho_{b}\left(v, r_{\star}\right)+\beta \varrho_{b}\left(r_{n_{k_{l}}+1}, r_{n_{k_{l}}}\right) \\
& +\frac{\gamma}{b^{2}} \varrho_{b}\left(r_{\star}, r_{n_{k_{l}}}\right)+\frac{\delta}{b} \varrho_{b}\left(r_{n_{k_{l}}+1}, r_{\star}\right)+\frac{\tau}{b} \varrho_{b}\left(v, r_{n_{k_{l}}}\right) .
\end{aligned}
$$

Taking limit as $k \longrightarrow \infty$ in above, we get

$$
\begin{aligned}
& \varrho_{b}\left(v, r_{\star}\right) \leq \alpha \varrho_{b}\left(v, r_{\star}\right)+\frac{\tau}{b} \varrho_{b}\left(v, r_{\star}\right), \\
& \varrho_{b}\left(v, r_{\star}\right) \leq \alpha \varrho_{b}\left(v, r_{\star}\right)+\tau \varrho_{b}\left(v, r_{\star}\right) .
\end{aligned}
$$

It implies

$$
(1-(\alpha+\tau)) \varrho_{b}\left(v, r_{\star}\right) \leq 0 .
$$

If $\alpha+\tau=1$, then (19) implies $\beta=\gamma=\tau=0$ which implies $\alpha=1$, a contradiction, so

$$
\varrho_{b}\left(v, r_{\star}\right)=0 \text { implies } v=r_{\star} .
$$

Hence,

$$
\varrho_{b}\left(r_{\star}, T r_{\star}\right)=\varrho_{b}(R, S) .
$$

Similarly, we can prove

$$
\varrho_{b}\left(s_{\star}, T s_{\star}\right)=\varrho_{b}(R, S) .
$$

Now, let $r \in R, s \in S$, such that

$$
\varrho_{b}(r, \operatorname{Tr})=\varrho_{b}(R, S),
$$

and

$$
\varrho_{b}(s, T s)=\varrho_{b}(R, S) .
$$

If $\varrho_{b}(r, s)>\varrho_{b}(R, S)$, then

$$
\begin{aligned}
\varrho_{b}(r, s)< & \alpha \varrho_{b}(r, r)+\beta \varrho_{b}(s, s)+\frac{\gamma}{b^{2}} \varrho_{b}(r, s)+\frac{\delta}{b} \varrho_{b}(r, s) \\
& +\frac{\tau}{b} \varrho_{b}(r, s),
\end{aligned}
$$

$$
\varrho_{b}(r, s)<\gamma \varrho_{b}(r, s)+\delta \varrho_{b}(r, s)+\tau \varrho_{b}(r, s) .
$$

It implies

$$
(1-(\gamma+\delta+\tau)) \varrho_{b}(r, s)<0,
$$

which further implies

$$
\varrho_{b}(r, s)<0,
$$

a contradiction. So, $\varrho_{b}(r, s)=\varrho_{b}(R, S)$. This completes the proof.

Theorem 21. Let $R$ and $S$ be two nonempty subsets of $\left(P, \varrho_{b}\right)$, such that at least one of $R$ and $S$ is a bounded subset of $P$ and $(R, S)$ is a compact weak proximal pair. Let $T: R \cup S \longrightarrow R \cup S$ be a new type of generalized Hardy and Roger's proximal cyclic contractive mapping and assume that

(i) For each $r \in R_{0}$, $\operatorname{Tr} \in S_{0}$, and for each $s \in S_{0}$, Ts $\in R_{0}$,

(ii) If $\left(r_{n}\right)$ and $\left(s_{n}\right)$ are two bounded sequences in $R$ and $S$, respectively, such that $\left(\varrho_{b}\left(x_{n}, y_{n}\right)\right)$ converges to $\varrho_{b}(R, S)$, then $\lim _{n \longrightarrow \infty} \varrho_{b}\left(r_{n}, r_{n+1}\right)=0$.

Then, there exist $r \in R$ and $s \in S$, such that $\varrho_{b}(r, \operatorname{Tr})=$ $\varrho_{b}(R, S)$ and $\varrho_{b}(s, T s)=\varrho_{b}(R, S)$. Furthermore, $\varrho_{b}(r, s)=$ $\varrho_{b}(R, S)$.

Proof. Following Theorem 20, we can get the required result.

Corollary 22. If we take $b=1, \alpha=\beta=1 / 2$, and $\gamma=\delta=\tau=$ 0 in Theorem 21, we get Theorem 11.

\section{Some Fixed Points Results}

In this section, we derive some fixed points results from our main results.

Theorem 23. Let $\left(P, \varrho_{b}\right)$ be a boundedly compact b-metric space; then,

(i) A mapping T:P $\longrightarrow \wp(P)$, such that for $q_{1}, q_{2} \in P$, there exist $p_{1} \in T q_{1}$ and $p_{2} \in T q_{2}$, such that

$$
\begin{aligned}
\varrho_{b}\left(p_{1}, p_{2}\right)< & \alpha \varrho_{b}\left(p_{1}, q_{1}\right)+\beta \varrho_{b}\left(p_{2}, q_{2}\right)+\frac{\gamma}{b^{2}} \varrho_{b}\left(q_{1}, q_{2}\right) \\
+ & \frac{\delta}{b} \varrho_{b}\left(p_{1}, q_{2}\right)+\frac{\tau}{b} \varrho_{b}\left(p_{2}, q_{1}\right), \mathrm{s} .
\end{aligned}
$$

$$
\left.\begin{array}{l}
\text { For } q_{1} \neq q_{2} \\
\text { And } \\
\varrho_{b}\left(p_{1}, p_{2}\right) \leq \alpha \varrho_{b}\left(p_{1}, q_{1}\right)+\beta \varrho_{b}\left(p_{2}, q_{2}\right)+ \\
\frac{\delta}{b} \varrho_{b}\left(p_{1}, q_{2}\right)+\frac{\tau}{b} \varrho_{b}\left(p_{2}, q_{1}\right)
\end{array}\right\},
$$




$$
\begin{aligned}
& \text { For } q_{1}=q_{2}, \text { where } \\
& \qquad \alpha+\beta+\gamma+2 \tau=1, \beta \neq 1, \gamma+\delta+\tau<1,
\end{aligned}
$$

$\alpha, \beta, \gamma, \delta, \tau \in \mathbb{R}^{+}$.

(ii) If $\left(p_{n}\right)$ is a bounded sequence in $P$, then $\lim _{n \longrightarrow \infty}\left(p_{n}, p_{n+1}\right)=0$.

Then, Fix $(T)$ (set of fixed points of $T$ ) is nonempty

Theorem 24. Let $\left(P, \varrho_{b}\right)$ be a boundedly compact b-metric space; then,

(i) A mapping $T: P \longrightarrow P$, such that for all $q_{1}, q_{2} \in P$ with $q_{1} \neq q_{2}$,

$$
\begin{aligned}
\varrho_{b}\left(T q_{1}, T q_{2}\right)< & \alpha \varrho_{b}\left(T q_{1}, q_{1}\right)+\beta \varrho_{b}\left(T q_{2}, q_{2}\right)+\frac{\gamma}{b^{2}} \varrho_{b}\left(q_{1}, q_{2}\right) \\
& +\frac{\delta}{b} \varrho_{b}\left(T q_{1}, q_{2}\right)+\frac{\tau}{b} \varrho_{b}\left(T q_{2}, q_{1}\right),
\end{aligned}
$$

where

$$
\alpha+\beta+\gamma+2 \tau=1, \beta \neq 1, \gamma+\delta+\tau<1,
$$

$\alpha, \beta, \gamma, \delta, \tau \in \mathbb{R}^{+}$.

(ii) If $\left(p_{n}\right)$ is a bounded sequence in $P$, then $\lim _{n \longrightarrow \infty}\left(p_{n}, p_{n+1}\right)=0$

Then, Fix $(T)$ is singleton.

\section{Conclusion}

We presented a new type of generalized multivalued Hardy and Roger's proximal contractive and proximal cyclic contractive mappings in $b$-metric spaces and developed results for the existence of best proximity points. Furthermore, we have derived results for the existence and uniqueness of best proximity points for new type of generalized Hardy and Roger's proximal contractive and proximal cyclic contractive mappings. Our results are the generalization of the results already existing in literature.

\section{Data Availability}

The data used to support the findings of this study are available from the corresponding author upon request.

\section{Conflicts of Interest}

The authors declare that they have no conflicts of interest.

\section{References}

[1] S. Banach, "Sur les opérations dans les ensembles abstraits et leur application aux équations intégrales," Fundamenta Mathematicae, vol. 3, pp. 133-181, 1922.

[2] R. P. Agarwal, N. Hussain, and M. A. Taoudi, "Fixed point theorems in ordered Banach spaces and applications to nonlinear integral equations," Abstract and Applied Analysis, vol. 2012, Article ID 245872, 15 pages, 2012.
[3] V. Berinde, Iterative Approximation of Fixed Points, Springer, Berlin, Germany, 2007.

[4] M. Delfani, A. Farajzadeh, and C. F. Wen, "Some fixed point theorems of generalized $F_{t}$-contraction mappings in b-metric spaces," Journal of Nonlinear and Variational Analysis, vol. 5, no. 4, pp. 615-625, 2021.

[5] P. Hu and F. Gu, "Some fixed point theorems of $\lambda$-contractive mappings in Menger PSM-spaces," Journal of Nonlinear Functional Analysis, vol. 2020, Article ID 8447435, 8 pages, 2020.

[6] S. K. Malhotra, S. Prakash, and S. Shukla, "A generalization of Nadler theorem in cone b-metric spaces over Banach algebras," Communications in Optimization Theory, vol. 4, Article ID 10, 2019.

[7] T. Suzuki, "A generalized Banach contraction principle that characterizes metric completeness," Proceedings of the American Mathematical Society, vol. 136, no. 5, pp. 1861-1869, 2008.

[8] T. Suzuki, "A new type of fixed point theorem in metric spaces," Nonlinear Analysis: Theory, Methods \& Applications, vol. 71, no. 11, pp. 5313-5317, 2009.

[9] M. Edelstein, "On fixed and periodic points under contractive mappings," Journal of the London Mathematical Society, vol. s1-37, no. 1, pp. 74-79, 1962.

[10] A. Meir and E. Keeler, "A theorem on contraction mappings," Journal of Mathematical Analysis and Applications, vol. 28, no. 2, pp. 326-329, 1969.

[11] S. Reich, "A fixed point theorem, Atti della Accademia Nazionale dei Lincei. Classe di Scienze Fisiche, Matematiche e Naturali," Rendiconti, vol. 51, no. 1-2, pp. 26-28, 1971.

[12] G. E. Hardy and T. D. Rogers, "A generalization of a fixed point theorem of Reich," Canadian Mathematical Bulletin, vol. 16, no. 2, pp. 201-206, 1973.

[13] S. NadlerJr., "Multi-valued contraction mappings," Pacific Journal of Mathematics, vol. 30, no. 2, pp. 475-488, 1969.

[14] J. B. Prolla, "Fixed-point theorems for set-valued mappings and existence of best approximants," Numerical Functional Analysis and Optimization, vol. 5, no. 4, pp. 449-455, 1983.

[15] I. A. Bakhtin, "The contraction mapping principle in quasi metric spaces," Functional Analysis, vol. 30, pp. 26-37, 1989.

[16] S. Czerwik, "Contraction mappings in b-metric spaces," Acta mathematica et informatica universitatis ostraviensis, vol. 1, no. 1, pp. 5-11, 1993.

[17] K. Fan, "Extensions of two fixed point theorems of F. E. Browder," Mathematische Zeitschrift, vol. 112, no. 3, pp. 234-240, 1969.

[18] V. M. Sehgal and S. P. Singh, "A generalization to multifunctions of Fan's best approximation theorem," Proceedings of the American Mathematical Society, vol. 102, no. 3, pp. 534-537, 1988.

[19] A. Abkar and M. Gabeleh, "A best proximity point theorem for Suzuki type contraction nonself mappings," Fixed Point Theory, vol. 14, no. 2, pp. 281-288, 2013.

[20] S. Sadiq Basha, "Best proximity point theorems," Journal of Approximation Theory, vol. 163, no. 11, pp. 1772-1781, 2011.

[21] S. S. Basha, "Best proximity point theorems for some special proximal contractions," Numerical Functional Analysis and Optimization, vol. 40, no. 10, pp. 1182-1193, 2019.

[22] S. S. Basha and N. Shahzad, "Best proximity point theorems for generalized proximal contraction," Fixed Point Theory and Applications, vol. 42, no. 1, pp. 1-9, 2012.

[23] N. Hussain, A. Latif, and P. Salimi, "Best proximity point results for modified Suzuki $(\alpha-\psi)$-proximal contractions," Fixed Point Theory and Applications, vol. 1, pp. 1-16, 2014. 
[24] W. A. Kirk, P. S. Srinivasan, and P. Veeramani, "Fixed points for mappings satisfying cyclical contractive conditions," Fixed Point Theory, vol. 4, no. 1, pp. 79-89, 2003.

[25] A. Anthony and P. Veeramani, Proximal pointwise contraction, Topology and its Applications, vol. 156, no. 18, pp. 2942-2948, 2009.

[26] G. Hiranmoy, E. Karapınar, and L. K. Dey, "Best proximity point results for contractive and cyclic contractive type mappings," Numerical Functional Analysis and Optimization, vol. 2021, Article ID 1933518, 16 pages, 2021.

[27] G. Hiranmoy, L. K. Dey, and T. Senapti, "On Kannan type contractive mappings," Numerical Functional Analysis and Optimization, vol. 39, no. 13, pp. 1466-1476, 2018. 